IFN- $\alpha / \beta$ production by RIG-I-deficient plasmacytoid dendritic cells was essentially normal after NDV infection. In these cells, TLR instead mediated the induction of IFN- $\alpha / \beta$.

Thus, RIG-I seems to induce IFN- $\alpha / \beta$ production in several cell types, but TLRs remain the key pathway for innate recognition of RNA viruses in plasmacytoid dendritic cells. The use of these two pathways in different cell types may reflect adaptation of host defense to different pathogens.

Earlier this year, Foy et al. and Breiman et al. found that HCV has developed a mechanism to counteract RIG-I ${ }^{2,3}$. They traced this mechanism to the HCV nonstructural protease 3/4a (NS3/4a). When expressed in cell cultures with RIG-I, NS3/4a could prevent RIG-I from inducing IFN- $\beta$.

Breiman et al. showed that overexpression of the cytosolic kinases IKKع or TBK1 involved in the activation of IFN- $\beta$ could circumvent the $\mathrm{NS} 3 / 4 a-$ mediated block in IFN- $\beta$ induction. Both groups concluded that RIG-I is not a proteolytic substrate for NS3/4a, placing the ㅇ HCV block somewhere between RIG-I and IKKe, perhaps acting upon an unidentified adaptor protein (Fig. 1).

Foy et al. went on to harness this information to generate a small peptide that inhibits the active site of the NS3/4a protease. The peptide inhibitor enhanced the IFN- $\beta$ response and decreased HCV replication in cell culture. Similar inhibition of HCV replication was observed in cells that overexpressed IKK $\varepsilon^{3}$.

These results suggest that antagonizing NS3/4a proteolytic activity in HCV infected individuals may increase the antiviral immune response, hopefully limiting the ability of this virus to establish a persistent infection.

Over the last few years, TLR-dependent signaling pathways have stood in the spotlight of innate immunity, and deservedly so, given the importance and diversity of this system in pathogen recognition. The characterization of multiple receptors and cytoplasmic adaptor proteins required for TLR signaling has facilitated the discovery of additional pathways, such as RIG-I, which function independently of TLR but use overlapping cytoplasmic signaling cascades. RIG-I, for instance, operates in the cell cytoplasm, whereas TLRs are localized at the cell surface or in endosomal compartments.

The plasmacytoid dendritic cell, or natural IFN-producing cell as it is known in humans, appears to be the major IFN- $\alpha / \beta$-producing cell type. This may be because of the high level of expression of TLR7 and TLR9 and constitutive expression of IRF7, a downstream effector necessary for the type I IFN response ${ }^{5}$. Additionally, plasmacytoid dendritic cells retain TLR ligands for extended periods of time in early endosomal compartments, perhaps allowing for sustained signaling ${ }^{6}$.

So why don't all cell types have the capability to respond with the vigor of a plasmacytoid dendritic cell when confronted with an invading pathogen? Perhaps, more is not always better. A growing body of evidence indicates that dysregulated IFN- $\alpha / \beta$ production leads to autoimmune disorders, a price paid for too vigorous of a response ${ }^{7}$.
This 'new' class of RNA helicase-CARD domain proteins shows functional diversity, as indicated by studies of a homolog of mouse RIG-I known as Helicard. Helicard operates during apoptosis, is a substrate for caspases, and increases DNA degradation through its helicase domain ${ }^{8}$. Another link between cell-death pathways and the innate response to virus is that the Fas-associated death domain-containing protein is required in some cells for the induction of IFN- $\alpha / \beta$ by doublestranded $\mathrm{RNA}^{9}$. It is unclear what regulates the balance between cell survival and death pathways by this family of helicase-CARD proteins.

The emergence of RIG-I as a conduit between recognition of viral nucleic acid and the innate immune response adds insight into how these various signaling pathways interconnect. More practically, these results provide a new opportunity to modulate the interferon pathway in individuals infected with HCV or other pathogens ${ }^{10,11}$.

1. Kato, H. et al. Immunity 23, 19-28 (2005).

2. Foy, E. et al. Proc. Natl. Acad. Sci. USA 102, 2986 29891 (2005).

3. Breiman, A. et al. J. Virol. 79, 3969-3978 (2005)

4. Yoneyama, M. et al. Nat. Immunol. 5, 730-737 (2004).

5. Liu, Y.J. Annu. Rev. Immunol. 23, 275-306 (2005).

6. Honda, K. et al. Nature 434, 1035-1040 (2005).

7. Theofilopoulos, A.N., Baccala, R., Beutler, B. \& Kono, D.H. Annu. Rev. Immunol. 23, 307-336 (2005).

8. Kovacsovics, M. et al. Curr. Biol. 12, 838-843 (2002).

9. Balachandran, S., Thomas, E. \& Barber, G.N. Nature 432, 401-405 (2004)

10. Zhong, J. et al. Proc. Natl. Acad. Sci. USA 102, 9294 9299 (2005)

11. Sumpter, R. Jr. et al. J. Virol. 79, 2689-2699 (2005).

\title{
Hatching a drug
}

Human monoclonal antibodies have hatched from chicken eggs (Nat. Biotechnol. 23, 1159-1169). Generating antibodies in eggs could prove more efficient than more traditional methods of cell culture.

To generate antibody-producing eggs, Lei Zhu et al. created an antibody transgene hooked up to regulatory elements for albumin, which is expressed at high levels in eggs. The investigators then transfected that gene into chicken embryonic stem cells; these cells, in turn, were used to create chickens that pumped out antibodies in the tubular gland cells of the oviduct (cells derived from stem cells are labeled in green (GFP), antibody in red and DNA in blue).

The egg-derived antibodies largely live up to the quality standards of antibodies made in cultured cells - having, for instance, similar affinity to antigen. Some 17 monoclonal antibody-based drugs have been approved by the US Food and Drug Administration in the last 20 years and many more are on deck.

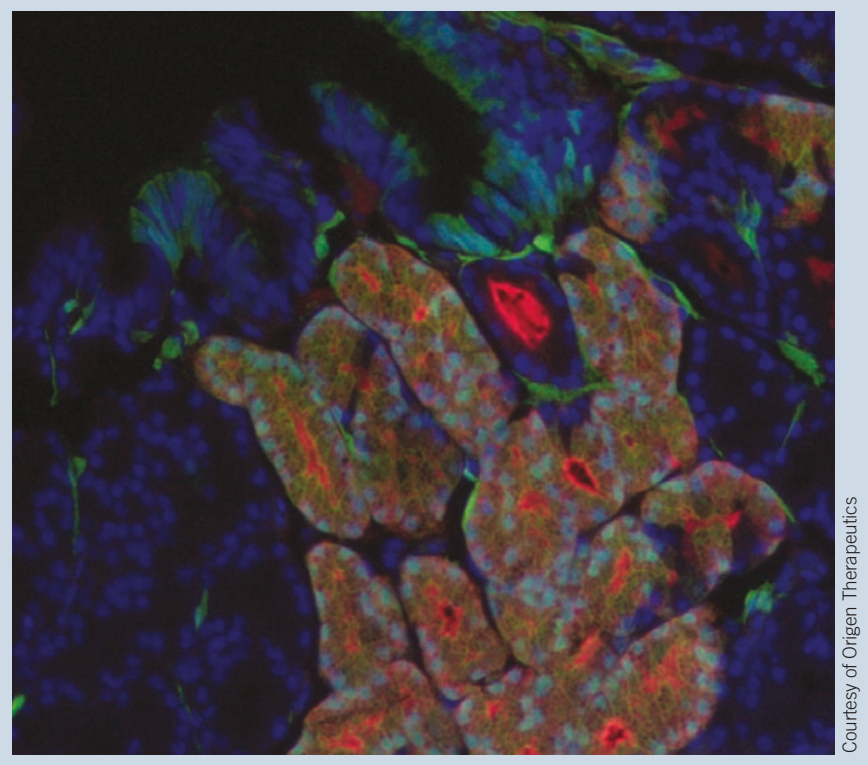

Charlotte Schubert 